\title{
FREEWAY WORK ZONE TRAFFIC STATE ESTIMATION WITH FAULT DIAGNOSIS
}

\author{
Shuming $\mathrm{Du}^{1}$ and Saiedeh Razavi ${ }^{2}$ \\ ${ }^{1}$ Department of Civil Engineering, McMaster University, Hamilton, Ontario, Canada \\ ${ }^{2}$ Chair in Heavy Construction, Department of Civil Engineering, McMaster University, \\ Hamilton, Ontario, Canada
}

\begin{abstract}
Freeway work zone can cause disruption to local traffic and have adverse impacts on mobility and safety of road users and those who work in the work zone. To ensure an effective traffic management strategy, it is essential to accurately and instantaneously estimate the traffic states at the work zone area. While many traffic state estimation methods are proposed by previous studies, few of them consider the occurrence of freeway sensor faults, which may result in a large deviation in state estimation and potentially lead to an inappropriate traffic management strategy. To overcome the impacts of sensor faults and provide accurate traffic state estimation, this study presents an approach using sensor fault diagnosis for traffic state estimation at freeway work zone area. Considering the capacity drop, the switching mode model with Kalman filter was used to estimate the traffic states. With the analysis of the density residuals generated by traffic sensors and probes, the fault diagnosis can detect the type of sensor faults and reconfigure the estimation model. The proposed system is implemented and evaluated in traffic simulator SUMO under a realistic freeway work zone environment. The results show that the developed system can accurately identify the type of fault in short time. An accurate traffic state estimation is provided and fairly maintained under fault-free and sensor-fault scenarios respectively.
\end{abstract}

\section{Introduction}

With the aging of freeway infrastructure, numerous construction work zones are added to freeway for projects such as barrier replacement, road resurfacing and road expansion every year. These work zones with lane closure can easily lead to traffic congestion and cause high safety risks and costs associated with wasted time and fuel. In 2014, 888 million hours and over 310 million gallons were wasted due to construction work zones (FHWA, 2014). To reduce the impacts of freeway work zones and mitigate the traffic congestion, many traffic management strategies are developed. The variable speed limit control was designed to dynamically change the speed limits upstream of a work zone so as to restrict the traffic inflow and maintain the maximum work zone throughput (Papamichail et al., 2018; Du et al., 2019). A lane changing control strategy for work zone was proposed to reduce the lane merging conflicts and improve safety near work zone area (Qi et al., 2017). Hou et al. (2015) designed a traffic flow forecasting system to analyze the impact of planned work zone. However, an accurate traffic state estimation is essential to ensure the effectiveness of the traffic management strategies.

To provide an accurate traffic state estimation, various studies have utilized the freeway stationary sensors or a combination of stationary sensors and connected vehicles which act as probe sensors. The performances of traffic state estimation were compared between a sliding mode observer and an extended Kalman filter by using the loop detectors (Majid et al., 2015). By estimating the penetration rates of connected vehicle, the traffic density was calculated under mixed connected vehicles and conventional vehicle environments (Bekiaris-Liberis et al., 2016). Chu et al. (2018) developed the stochastic Lagrangian traffic flow model to estimate and predict the traffic congestion using lagrangian traffic data. The smart work zone was also built to acquire a better performance of queue and travel time estimation ( $\mathrm{Li}$ et al., 2018). However, the aforementioned studies greatly relied on fault-free sensors which can consistently detect the traffic condition without disruption. In reality, a large part of data samples is missing due to sensor malfunction. It is reported that only $60 \%$ of the sensor network in California can provide reliable traffic measurement (Rajagopal et al., 2007). With the absence of reliable traffic measurement, the accuracy of traffic state estimation is greatly affected.

Since the sensor faults have detrimental effects on traffic data quality, many researchers have proposed a number of methods to detect the sensor faults and impute the missing data. The matrix and tenser based methods were designed by Asif et al. (2016) to learn traffic patterns so as to fill in the gap of the speed data. Chen et al. (2003) developed the diagnostics algorithm by using the time series of data samples based on linear regression method to estimate missing values. A modified $\mathrm{k}$-nearest neighbour method and genetic algorithms were also utilized to improve the performance of the data imputation in missing traffic 
data by Tak et al. (2016) and Tang et al. (2015) respectively. A better traffic state estimation is achieved in the previous studies by considering faulty sensors and missing samples, however, the designed diagnosis algorithms demand large historical data samples which are not always available especially in the cases of short-term work zones. Meanwhile, the previous diagnosis methods cannot provide sensor fault detection and adjust the state estimation algorithms online. Consequently, this makes it difficult to incorporate aforementioned traffic state estimation algorithms in traffic management strategies as real-time traffic condition is needed to make appropriate decisions.

To overcome the impacts of sensor faults on the traffic state estimation and provide accurate fault diagnosis and traffic condition, this study focuses on developing a traffic state estimation method with sensor fault diagnosis algorithm. The study includes: 1) designing the traffic state estimation model which can accurately generate the traffic condition using the stationary sensors and probe sensors; 2) developing a fault diagnosis algorithm which can detect the types of sensor faults and reconfigure the state estimation model; 3) implementing and evaluating the designed system in traffic simulator SUMO under realistic freeway work zone environment.

The rest of this paper is organized as follows. First, the system framework is presented. Then the traffic flow model is developed using the switching mode model with capacity drop. With the developed traffic flow model, the Kalman filter is utilized to estimate the traffic states. Following that, the fault diagnosis algorithm is built using the residual analysis. Afterwards, the effectiveness of the proposed system is evaluated in a traffic simulator environment. The conclusions and future work of this study are discussed in the end.

\section{Methodology}

\section{System Framework}

The proposed system mainly consists of three components: the traffic flow model, fault diagnosis and Kalman filter as shown in Figure 1.

In this study, connected vehicles are used as probe sensors in addition to other traffic sensors that can be used in fixed locations. The traffic measurements such as vehicle speed and traffic flow rate, which are detected by these two types of sensors, are sent to the traffic flow model, to estimate the traffic states and to be used for one-step state prediction. The capacity drop that further decreases the work zone capacity is also considered in the designed traffic flow model. The Kalman filter is used to improve the accuracy of the estimation by using one-step prediction and the traffic measurements collected by sensors. Then the improved traffic state estimation is generated and also sent back to the traffic flow model to be used in the next time step estimation. During the process of state estimation, the fault diagnosis algorithm collects the traffic measurements from two types of sensors to determine whether a sensor fault occurs and to reconfigure the estimation model in the presence of a sensor fault.

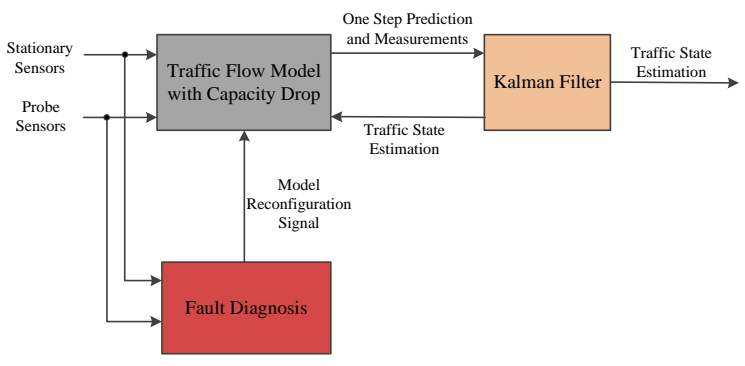

Figure 1: System framework

\section{Traffic Flow Model}

Freeway work zone with lane closure can cause the loss of the road capacity. As a result, a queue easily forms upstream of a work zone when the traffic demand is larger than the work zone capacity. To estimate traffic states such as traffic density and speed near work zone area, traffic sensors and connected vehicles are used at work zone area as shown in Figure 2.

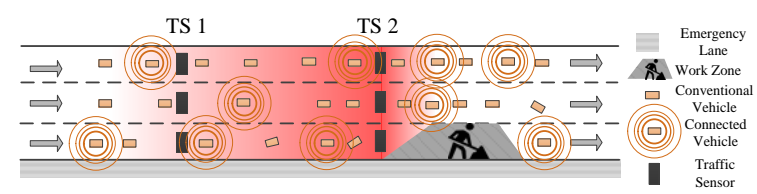

Figure 2: Layout of freeway work zone

Due to construction work zone, one of three lanes is closed in Figure 2. The mixed traffic flow which has conventional vehicles and connected vehicle travels from the left side to right side. In this study, connected vehicles are considered as probe sensors due to their ability to transmit positions and speeds. We assumed the drivers' behavior for conventional and connected vehicles is the same. Two traffic sensors stations TS 1 and TS 2 are located next to each other near work zone area. Because of the loss of road capacity, traffic congestion easily occurs when the demand exceeds the work zone capacity and a queue forms the upstream of the work zone.

Since traffic management strategies such as variable speed limit control and lane merging control are mainly implemented $1 \mathrm{~km}$ to $2 \mathrm{~km}$ upstream of work zone (Yang et al., 2017; Qi et al., 2017), the traffic state estimation in this immediate upstream of work zone area is crucial. Therefore, this upstream area is studied and the layout of this part of freeway is shown in Figure 3. 


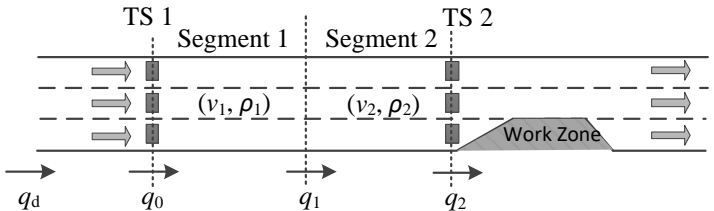

Figure 3: Layout of freeway segments

The section between TS 1 and TS 2 can be divided by several segments. Without the loss of generality, two segments with the same length are selected and the following proposed method can potentially apply to more segments. The traffic inflows of segment 1 and segment 2 are expressed as $q_{0}$ and $q_{1}$ respectively. $q_{d}$ represents the traffic demand and $q_{2}$ is the work zone throughput. The variable $v_{i}$ and $\rho_{i}$ where $i$ equals 1 or 2 are the average speed and density respectively in segment $i$ which need to be estimated.

The traffic density $\rho_{i}(k)$ in segment $i$ at time step $k$ can be updated using the conservation law (Lighthill and Whitham, 1955; Richards, 1956)

$$
\rho_{i}(k+1)=\rho_{i}(k)+\frac{\Delta T_{s}}{L}\left[q_{i-1}(k)-q_{i}(k)\right], i=1,2
$$

where $\Delta T_{s}$ is the sample time interval and $L$ is the length of segment. Under the assumption of triangular fundamental diagram (Newell, 1993), the traffic flow can be calculated as

$$
\left\{\begin{array}{l}
q_{0}(k)=\min \left\{q_{d}(k), q_{\max }, \omega\left(\rho_{j}-\rho_{1}\right)\right\} \\
q_{1}(k)=\min \left\{v_{1}(k) \rho_{1}(k), q_{\max }, \omega\left(\rho_{j}-\rho_{2}\right)\right\} \\
q_{2}(k)=\min \left\{v_{2}(k) \rho_{2}(k), q_{\mathrm{md}}\right\}
\end{array}\right.
$$

In Equation (2), $q_{\max }$ is the maximum flow rate while $q_{\mathrm{md}}$ is the maximum flow rate with capacity drop. $\omega$ and $\rho_{j}$, which represent the backward propagating wave speed and jam density respectively, are the parameters in fundamental diagram as shown in Figure 4.

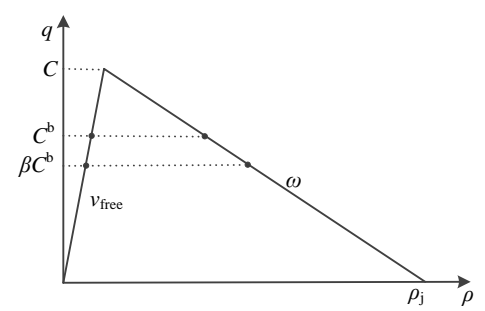

Figure 4: Triangular fundamental diagram

The two branches with the slopes of free flow speed $v_{\text {free }}$ and backward propagating wave speed $\omega$ show the free and congested statues respectively. The capacity $C$ of three-lane freeway is reduced to be $C^{b}=2 / 3 C$ due to one lane is closed at the work zone area. However, the reduced capacity $C^{b}$ decreases further when a queue forms upstream of a work zone (Hall et al., 1992; Chung et al., 2007). This is known as capacity drop phenomenon and the maximum flow rate with capacity drop $q_{\mathrm{md}}$ becomes $\beta C^{b}$ where $\beta$ is the capacity drop factor.

The traffic density evolution equation can be described by

$$
\boldsymbol{x}_{k+1}=\boldsymbol{A}_{k} \boldsymbol{x}_{k}+\boldsymbol{B}_{k} \boldsymbol{u}_{k}+\boldsymbol{\varepsilon}_{k}
$$

where $\boldsymbol{x}_{k}=\left[\rho_{1, k}, \rho_{2, k}\right]^{\mathrm{T}}$ is the state vector, $\boldsymbol{u}_{k}$ is the system input and $\boldsymbol{\varepsilon}_{k}$ is the system process noise assumed to be zero-mean Gaussian signal with the covariance matrix $\boldsymbol{Q}_{k}=E\left[\boldsymbol{\varepsilon}_{k} \boldsymbol{\varepsilon}_{k}^{\mathrm{T}}\right] . \boldsymbol{A}_{k}$ and $\boldsymbol{B}_{k}$ are system matrices.

Since the nonlinearity introduced by $\min$ function in Equation (2) makes it challenging to estimate the traffic state, the switching mode model is developed based on the traffic status (Munoz et al., 2003). This developed model can eliminate the nonlinearity and establish linear density evolution equations under different traffic statuses. The fixed head queue is considered in this study, while the tail of queue can move towards either upstream or downstream of work zone. Therefore, the status of the segment 1 and segment 2 can fall in the set $\{\mathrm{FF}, \mathrm{FCu}, \mathrm{CCu}, \mathrm{CCd}$, $\mathrm{FCd}$ \} where the first two letters indicates the status of segment 1 and segment 2 . The letter $u$ and $d$ represent the moving direction of the tail of the queue. $\mathrm{F}$ and $\mathrm{C}$ signify the free status and congested status respectively.

By differentiating the traffic status, only one value is selected from the min function in Equation (2) such that the nonlinearity is eliminated. The Equation (4) (8) show the density evolution of status FF, FCu, $\mathrm{CCu}, \mathrm{CCd}$ and $\mathrm{FCd}$ respectively.

$$
\begin{aligned}
& {\left[\begin{array}{c}
\rho_{1, k+1} \\
\rho_{2, k+1}
\end{array}\right]=\left[\begin{array}{cc}
1-\frac{\Delta T_{s}}{L} v_{1, k} & 0 \\
\frac{\Delta T_{s}}{L} & 1-\frac{\Delta T_{s}}{L} v_{2, k}
\end{array}\right]\left[\begin{array}{c}
\rho_{1, k} \\
\rho_{2, k}
\end{array}\right]+\left[\begin{array}{c}
\frac{\Delta T_{s}}{L} q_{d, k} \\
0
\end{array}\right]+\boldsymbol{\varepsilon}_{k}} \\
& {\left[\begin{array}{l}
\rho_{1, k+1} \\
\rho_{2, k+1}
\end{array}\right]=\left[\begin{array}{cc}
1 & \frac{\Delta T_{s}}{L} \omega \\
0 & 1-\frac{\Delta T_{s}}{L} \omega
\end{array}\right]\left[\begin{array}{l}
\rho_{1, k} \\
\rho_{2, k}
\end{array}\right]+\left[\begin{array}{l}
\frac{\Delta T_{s}}{L}\left(q_{d, k}-\omega \rho_{j}\right) \\
\frac{\Delta T_{s}}{L}\left(\omega \rho_{j}-q_{\mathrm{md}}\right)
\end{array}\right]+\boldsymbol{\varepsilon}_{k}}
\end{aligned}
$$

$$
\left[\begin{array}{c}
\rho_{1, k+1} \\
\rho_{2, k+1}
\end{array}\right]=\left[\begin{array}{cc}
1-\frac{\Delta T_{s}}{L} \omega & \frac{\Delta T_{s}}{L} \omega \\
0 & 1-\frac{\Delta T_{s}}{L} \omega
\end{array}\right]\left[\begin{array}{c}
\rho_{1, k} \\
\rho_{2, k}
\end{array}\right]+\left[\begin{array}{c}
0 \\
\frac{\Delta T_{s}}{L}\left(\omega \rho_{j}-q_{\mathrm{md}}\right)
\end{array}\right]+\boldsymbol{\varepsilon}_{k}
$$




$$
\begin{aligned}
& {\left[\begin{array}{c}
\rho_{1, k+1} \\
\rho_{2, k+1}
\end{array}\right]=\left[\begin{array}{cc}
1 & \frac{\Delta T_{s}}{L} \omega \\
0 & 1-\frac{\Delta T_{s}}{L} \omega
\end{array}\right]\left[\begin{array}{l}
\rho_{1, k} \\
\rho_{2, k}
\end{array}\right]+\left[\begin{array}{l}
\frac{\Delta T_{s}}{L}\left(q_{d, k}-\omega \rho_{j}\right) \\
\frac{\Delta T_{s}}{L}\left(\omega \rho_{j}-q_{\mathrm{md}}\right)
\end{array}\right]+\boldsymbol{\varepsilon}_{k}} \\
& {\left[\begin{array}{l}
\rho_{1, k+1} \\
\rho_{2, k+1}
\end{array}\right]=\left[\begin{array}{cc}
1-\frac{\Delta T_{s}}{L} v_{1, k} & 0 \\
\frac{\Delta T_{s}}{L} v_{1, k} & 1
\end{array}\right]\left[\begin{array}{l}
\rho_{1, k} \\
\rho_{2, k}
\end{array}\right]+\left[\begin{array}{l}
\frac{\Delta T_{s}}{L} q_{d, k} \\
-\frac{\Delta T_{s}}{L} q_{\mathrm{md}}
\end{array}\right]+\boldsymbol{\varepsilon}_{k}}
\end{aligned}
$$

In the case of the speed estimation, since the connected vehicles in this study are only used as probe sensors, the average speed of connected vehicles is utilized to estimate the average speed in segment 1 and 2 .

As stated before, traffic sensors and connected vehicles are utilized to detect the traffic condition. Therefore the measurement equation can be designed as

$$
\boldsymbol{y}_{k}=\boldsymbol{C}_{k} \boldsymbol{x}_{k}+\boldsymbol{\zeta}_{k}
$$

where $\boldsymbol{y}_{k}$ is the sensor measurement with noise $\boldsymbol{\zeta}_{k}$ assumed to be zero-mean Gaussian noise with the covariance $\boldsymbol{R}_{k}=E\left[\boldsymbol{\zeta}_{k} \boldsymbol{\zeta}_{k}^{\mathrm{T}}\right] . \boldsymbol{C}_{k}$ is the system matrix. More specifically, Equation (9) can be written as

$$
\left[\begin{array}{c}
\frac{C o_{1, k}-C o_{2, k}}{L} \\
\frac{q_{0, k}}{v_{1, k}}
\end{array}\right]=\left[\begin{array}{ll}
1 & 1 \\
1 & 0
\end{array}\right]\left[\begin{array}{l}
\rho_{1, k} \\
\rho_{2, k}
\end{array}\right]+\boldsymbol{\zeta}_{k}
$$

In Equation (10), $C o_{1, k}$ and $C o_{2, k}$ are the traffic count from TS 1 and TS 2. Since the queue spills back towards upstream, the traffic flow rate detected by TS 1 , which is located upstream of segment 1 , is utilized to calculate the density of segment 1 via dividing by average speed in segment 1 .

Therefore, the traffic state estimation model can be derived by combining the Equation (3) and (9). However, there are some noises in both density evolution equation and measurement equation which have negative impacts on the accuracy of traffic state estimation. Thus, the Kalman filter discussed in the following section is used to improve the estimation accuracy.

\section{Kalman Filter for Traffic State Estimation}

The Kalman filter is a recursive estimation algorithm which consists of two steps: the prediction step and measurement update step (Bar-Shalom et al., 2001). The following equations are utilized to update the traffic state:

$$
\boldsymbol{x}_{k+1 / k}=\boldsymbol{A}_{k} \boldsymbol{x}_{k}+\boldsymbol{B}_{k} \boldsymbol{u}_{k}
$$

$$
\begin{gathered}
\boldsymbol{P}_{k+1 / k}=\boldsymbol{A}_{k} \boldsymbol{P}_{k} \boldsymbol{A}_{k}^{T}+\boldsymbol{Q}_{k} \\
\boldsymbol{K}_{k}=\boldsymbol{P}_{k+1 / k} \boldsymbol{C}_{k}^{T}\left(\boldsymbol{C}_{k} \boldsymbol{P}_{k+1 / k} \boldsymbol{C}_{k}^{T}+\boldsymbol{R}_{k}\right)^{-1} \\
\boldsymbol{x}_{k+1}=\boldsymbol{x}_{k+1 / k}+\boldsymbol{K}_{k}\left(\boldsymbol{y}_{k}-\boldsymbol{C}_{k} \boldsymbol{x}_{k+1 / k}\right) \\
\boldsymbol{P}_{k+1}=\left(\boldsymbol{I}-\boldsymbol{K}_{k} \boldsymbol{C}_{k}\right) \boldsymbol{P}_{k+1 / k}
\end{gathered}
$$

After the one-step state prediction $\boldsymbol{x}_{k+1 / k}$ and state covariance prediction $\boldsymbol{P}_{k+1 / k}$, the filter gain $\boldsymbol{K}_{k}$ is calculated. The prediction is corrected in Equation (14) by using the traffic measurement from traffic sensors and connected vehicles together with filter gain $\boldsymbol{K}_{k}$. Then the covariance is also updated in Equation (15). Thus the traffic state estimation can be updated every time step with the measurement.

\section{Fault Diagnosis Scheme}

The fault diagnosis scheme is developed to identify the type of sensor fault, and then reconfigure the traffic state estimation model based on the type of faults. This study focuses on the stationary sensor faults, which are TS 1 and TS 2 in Figure 2. There are many types of stationary sensor faults. For example, it is found the sensor malfunction with zero flow rate is the leading fault among all the loop detector failure (Chen et al., 2003). Therefore sensor fault that causes the zero flow rate with the presence of vehicles is mainly considered in this study. However, there are 2 traffic sensors stations located upstream of the work zone. The different combinations of faulty sensors are categorized as different types of faults shown in Table 1.

Table 1: Types of sensor faults

\begin{tabular}{|c|c|}
\hline Fault Number & Description \\
\hline Fault 1 & Only TS 1 is down \\
\hline Fault 2 & Only TS 2 is down \\
\hline Fault 3 & $\begin{array}{c}\text { Both TS } 1 \text { and TS } 2 \\
\text { are down }\end{array}$ \\
\hline
\end{tabular}

The fault diagnosis utilized the measurement from traffic sensors and connected vehicles to achieve the fault diagnosis. The process of the diagnosis is demonstrated in Figure 5.

It can be seen from Figure 5, the density estimation can be acquired from two ways. One is to use the measurement Equation (9) to fuse the measurement from both sources: stationary traffic sensors and connected vehicles. The other is to use the fundamental diagram in Figure 4 to calculate the density $\rho_{1, k}^{\prime}$ and $\rho_{2, k}^{\prime}$ via the average speed of connected vehicles. The estimated densities using 
connected vehicles alone act as the redundant information. Then the redundant information is fed into the block of residual calculation together with the density estimation from both sensors. After the residual calculation, the residual analysis and the model reconfiguration are performed as shown in Figure 6.

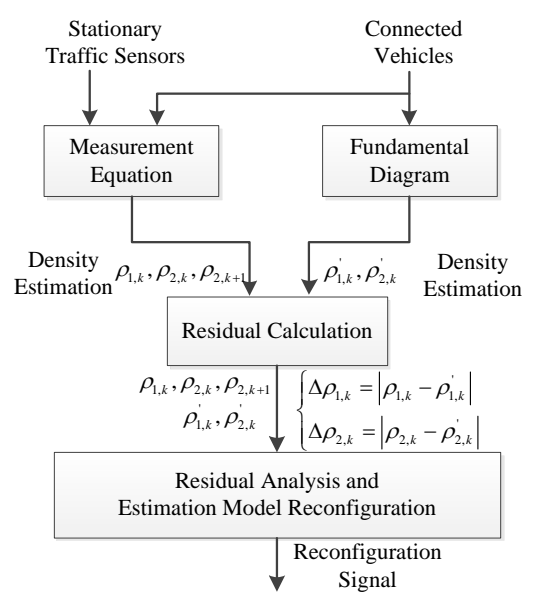

Figure 5: Fault diagnosis

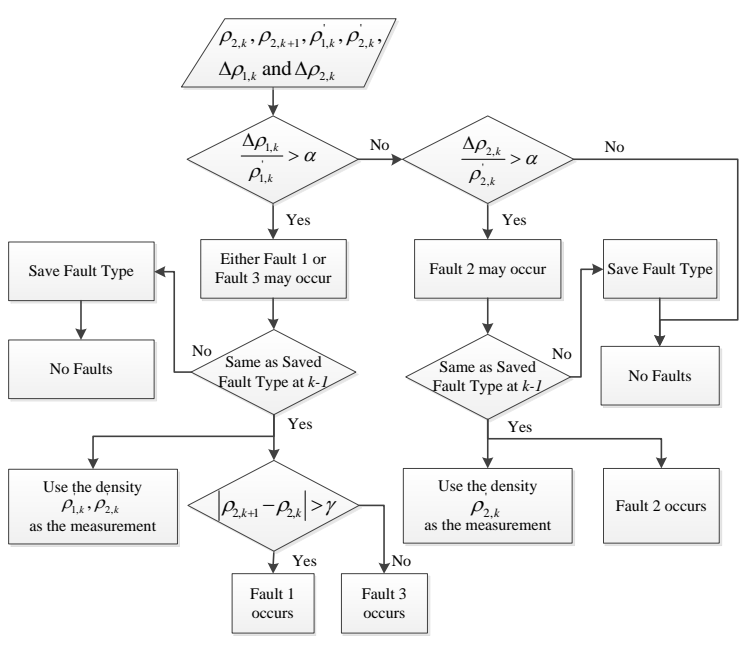

Figure 6: Residual analysis and model reconfiguration

As it can be seen from Figure 6, the identification of faults is achieved by the comparison between the density residual and the density estimated by connected vehicles, together with the comparison between current fault type and the saved fault type at $k$-1. $\alpha$ and $\gamma$ are selected as the thresholds to consider the model uncertainties and noises. Too large thresholds can delay the time when the fault is detected or even make the fault undetectable, while too small thresholds can cause false fault detection. Different combinations of the thresholds are tested in this study to select the appropriate values. The current fault type and saved fault type from last time step are compared to avoid false fault detection. When the type of the fault is detected and identified, the appropriate measure will be taken to reconfigure the estimation model. Though fault 1 and fault 3 can be successfully be distinguished, one more time step is required to confirm which fault occurs. However, both faults share the same reconfiguration action and there will be no time delay after the detection of either fault.

\section{Experiment and Results}

\section{Experiment Setup}

The proposed traffic state estimation for freeway work zone with fault diagnosis system is evaluated on

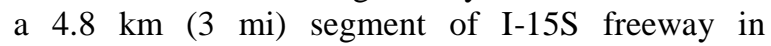
California, US. The selected segment has the maximum legitimate speed limit $113 \mathrm{~km} / \mathrm{h}(70 \mathrm{mi} / \mathrm{h})$. A construction work zone was setup starting from State PM (postmile) 40.2 and extending to State PM 37.6 on July 22, 2016. The selected segment has one lane closed due to this work zone. Two loop detectors located at State PM 41.6 and 40.1 act as the TS 1 and TS 2 in this study. As shown in Figure 7, the bold (blue) line is the segment selected to evaluate the proposed system. The thin (red) line inside the bold line represents the work zone area where one of three lanes is closed. The upper (purple) marker and bottom (yellow) mark are the positions of two loop detectors used in the experiment.

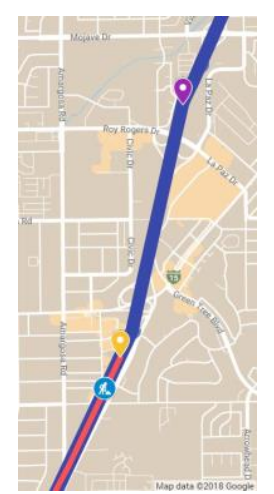

Figure 7: Traffic network with work zone

In this study, the traffic network was first built in the traffic simulator SUMO. Then the microscopic model was calibrated and validated using the real traffic data provided by California PeMS (Freeway Peformance Measurement System) database (PeMS, 2016).

After the traffic network was built, the fundamental diagram was calibrated using the generated traffic flow. The details of the calibration can be found in SUMO user manual (Krajzewicz et al., 2012). The capacity $C$, capacity drop factor $\beta$, jam density $\rho_{j}$, free flow speed $v_{\text {free }}$ and backward propagating wave speed $\omega$ are selected as $4800 \mathrm{veh} / \mathrm{h}, 0.94,270$ veh $/ \mathrm{km}, 108 \mathrm{~km} / \mathrm{h}$ and $21 \mathrm{~km} / \mathrm{h}$. According to the realistic traffic data statistics analysis (Bekiaris- 
Liberis, et al., 2016), the standard deviation of the noises of stationary sensors and probe sensors were selected as $25 \mathrm{veh} / \mathrm{h}$ and $3 \mathrm{~km} / \mathrm{h}$ respectively. All sensors are sampled every $30 \mathrm{~s}$. Both segment 1 and segment 2 have the length of $1.2 \mathrm{~km}(0.7 \mathrm{mi})$. The threshold $\alpha$ and $\gamma$ are selected as 0.8 and $5 \mathrm{veh} / \mathrm{km}$.

\section{Results}

To evaluate the effectiveness of the fault diagnosis scheme, 4 scenarios under different connected vehicle market penetration rates are designed: 1 ) no fault; 2) Fault 1; 3) Fault 2; 4) Fault 3. 20\% and 5\% are selected as the high and low connected vehicle market penetration rates respectively.

The simulation was run for $1 \mathrm{~h}$. The first 5 minutes is the warm-up period and simulation data for these 5 minutes is discarded. During the simulation, an artificial fault is created at time step $1400 \mathrm{~s}$ for all the scenarios. Due to the paper length limitations, only results under low connected vehicle market penetration rate $5 \%$ are presented for the residual analysis and fault detection in Figure 8, 9, 10 and 11.

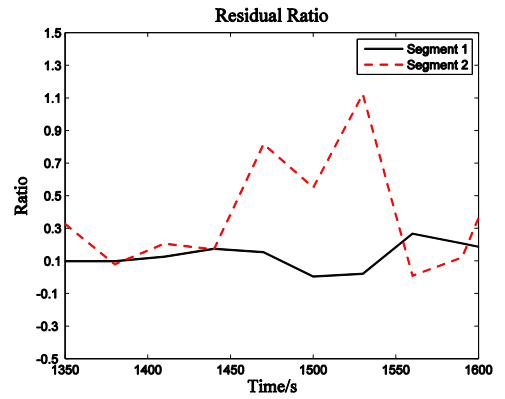

Figure 8: Residual analysis without fault

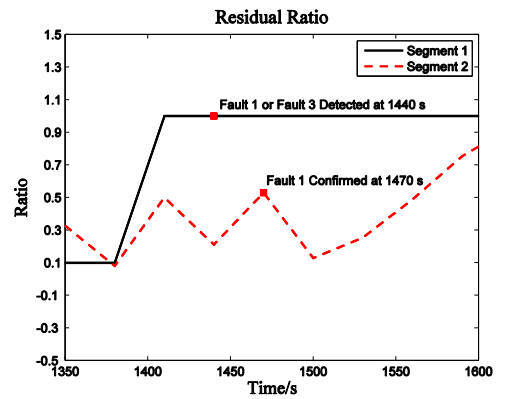

Figure 9: Residual analysis with fault 1

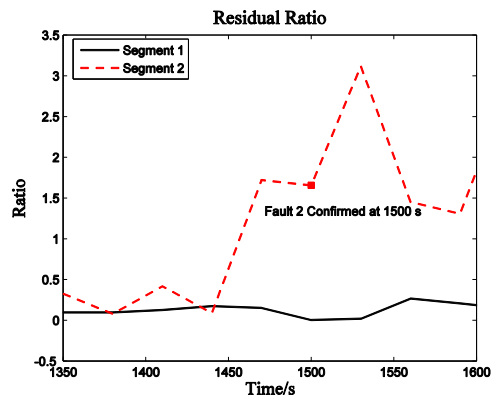

Figure 10: Residual analysis with fault 2

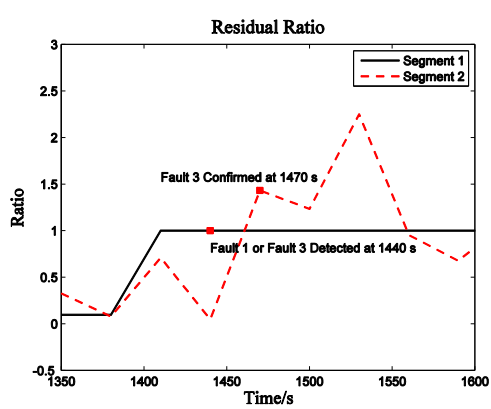

Figure 11: Residual analysis with fault 3

It can be seen from Figure 8 - 11 that all the faults have been successfully detected. In the scenario without any fault, the residual ratio in segment 1 is kept below 0.8 all the time before and after the artificial fault occurs. Although the residual ratio in segment 2 is larger than the threshold at some time steps, there is still no fault detected as no same fault type is detected between two consecutive time steps. The residual ratio in segment 2 exceeds the threshold some time because of the speed estimation error caused by low penetration rate and traffic merging disturbance at the immediate upstream of work zone. In Figure 9 and 11, either Fault 1 or 3 is detected at time step $1440 \mathrm{~s}$ because the fault type same as that at last sample time step $1410 \mathrm{~s}$ is detected. Though the specific type of the fault is confirmed one sample time interval later at the time step $1470 \mathrm{~s}$, the reconfiguration command is able to generate promptly. In the case of Fault 2, the sensor fault is detected at time step $1500 \mathrm{~s}$, which is later than the detection time of Fault 1 and Fault 3, as the density in segment 2 does not have a rapid change when the TS 2 fails. It requires some time for the fault diagnosis to identify the abnormal density increase in segment 2 . However, the results for density and speed estimation in the following part show that this delay does not have a significant impact on the accuracy of traffic state estimation under Fault 2 scenario.

To assess the accuracy of the traffic state estimation, the state estimation is compared with the ground truth as a reference using the Equation (16) RMSE (root mean square error). The ground truth can be acquired via built-in functionality in SUMO.

$$
R M S E=\sqrt{\frac{\sum\left(x_{i}-x_{i, r}\right)^{2}}{n}}
$$

where $x_{i}$ is the estimation and $x_{i, r}$ is the reference value. $\mathrm{n}$ is the total number of the measurement.

The results of traffic speed and density estimation are shown in Table 2, 3 and 4. Since the average speed is estimated using the connected vehicles which will not be affected by the faulty sensor, the RMSE results for speed estimation are only presented with different connected vehicle market penetration rates. 
Table 2: RMSE for speed estimation with different connected vehicle market penetration rates

\begin{tabular}{|c|c|c|}
\hline & \multicolumn{2}{|c|}{$\begin{array}{c}\text { Connected Vehicle Market } \\
\text { Penetration Rate }\end{array}$} \\
\hline Segment & $\mathbf{2 0 \%}$ & $\mathbf{5 \%}$ \\
\hline $\begin{array}{c}\text { Segment } 1 \\
(\mathrm{~km} / \mathrm{h})\end{array}$ & 3.8 & 6.7 \\
\hline $\begin{array}{c}\text { Segment } 2 \\
(\mathrm{~km} / \mathrm{h})\end{array}$ & 8.3 & 14.6 \\
\hline
\end{tabular}

Table 3: RMSE for density estimation with $20 \%$ connected vehicle market penetration rate

\begin{tabular}{|c|c|c|}
\hline & \multicolumn{2}{|c|}{ Density } \\
(Penetration Rate 20\%)
\end{tabular}

Table 4: RMSE for density estimation with 5\% connected vehicle market penetration rate

\begin{tabular}{|c|c|c|}
\hline & \multicolumn{2}{|c|}{ Density } \\
(Penetration Rate 5\%) \\
\hline Fault Type & Segment1 & Segment 2 \\
\hline $\begin{array}{c}\text { No Fault } \\
\text { (veh/km) }\end{array}$ & 11.4 & 10.4 \\
\hline $\begin{array}{c}\text { Fault 1 } \\
\text { (veh/km) }\end{array}$ & 14.1 & 19.3 \\
\hline $\begin{array}{c}\text { Fault 2 } \\
\text { (veh/km) }\end{array}$ & 12.1 & 15.4 \\
\hline $\begin{array}{c}\text { Fault 3 } \\
\text { (veh/km) }\end{array}$ & 15.2 & 19.3 \\
\hline
\end{tabular}

From Table 2, it can be seen that RMSEs are smaller in both segment 1 and segment 2 under high connected vehicle market penetration rate because generally more probe sensors can acquire more accurate estimation. Though speed estimation in segment 2 is larger than the estimation in segment 1, the overall speed estimations in both segments have a small error and a good performance.

As we can see from Table 3 and 4 , there is a small error (from $4 \mathrm{veh} / \mathrm{km}$ to $12 \mathrm{veh} / \mathrm{km}$ ) in the density estimation without any fault. One potential reason can be the length of segment 1 and 2 is slightly large. Longer segment will make the segment stay at mixed status between free flow and congestion for longer time. This mixed status may affect the switching mode model and potentially influence the accuracy of density estimation.

Table 3 and Table 4 show that Fault 1 and 3 have more impacts on the estimation than the impacts of Fault 2. Since both loop detectors are not used when TS 1 is down, more information of traffic condition from loop detectors is lost compared with Fault 2 scenario. Thus the performance of Fault 1 and 3 can be slightly worse. Meanwhile, only using the density measured by connected vehicles can also introduce some error because of the uncertainty in fundamental diagram.

The comparison of density estimation under high and low connected vehicle market penetration rates shows that higher penetration rate has better performance but the difference is not very significant. This can be attributed to the performance of speed estimation under different penetration rates.

In general, the results indicate that both the speed and density estimation provide a good accuracy of estimation. The performance is also fairly maintained under all types of faults especially when it is compared with the unusable estimation without fault diagnosis scheme.

\section{Conclusions and Future Work}

To improve the accuracy of estimating the traffic states for freeway work zone, a state estimation system with fault diagnosis is developed in this study. The switching mode model with the Kalman fitler can provide sufficient accuracy of the speed and density estimation. Three types of faults are successfully detected and identified. According to the detected faults, the fault diagnosis scheme can reconfigure the estimation model and a fairly good performance is maintained with the presence of sensor faults. The proposed system shows the ability to consistently provide accurate traffic state estimation for freeway work zone even with the sensor faults.

In this study, two segments were selected and only stationary sensor faults were considered. The impact of the length of segment on the state estimation was not analyzed. The communicate delay or bad samples from connected vehicles can also affect the accuracy of state estimation. In future work, more segments and faulty probe sensors will be studied and incorporated to achieve a better estimation.

\section{References}

Asif, M., Mitrovic, N., Dauwel, J., \& Jaillet, P. (2016). Matrix and tensor based methods for missing data estimation in large traffic netowrks. IEEE Transactions on Intelligent Transportation Systems, 17(7), pp. 1816-1815. 
Bar-Shalom, Y., Li, X. R., \& Kirubarajan, T. (2001). Estimation with Applications to Tracking and Navigation: Theory, Algorithms, and Software. New York: Wiley, 2001.

Bekiaris-Liberis, N., Roncoli, C., \& Papageorgiou, M. (2016). Highway traffic state estimation with mixed connected and conventional vehicles. IEEE Transactions on Intelligent Transportation Systems, 17(12), pp. 3484-3497.

Chen, C., Kwon, J., Rice, J., Skabardonis, A., \& Varaiya, P. (2003). Detecting Errors and Imputing Missing Data for Single-Loop Surveillance Systems. Transportation Research Record, 1855, pp. 160-167.

Chu, K., Saigal, R., \& Saitou, K. (2018). Real-time traffic prediction and probing strategy for Lagrangian traffic data. IEEE Transactions on Intelligent Transportation Systems, 20(2), pp. 497-506.

Chung, K., Rudjanakanoknad, J., \& Cassidy, M.J. (2007). Relation between traffic density and capacity drop at three freeway bottlenecks. Transportation Research Part B, 41, pp. 82-95.

Du, S., \& Razavi, S. (2019). Variable speed limit for freeway work zone with capacity drop using discrete-time sliding mode control. Journal of Computing in Civil Engineering, 33(2):04019001.

FHWA. (2014). "Work zone management program." $<$ https://ops.fhwa.dot.gov/wz/resources/ facts_stats.htm> (Feb. 13, 2018).

Hall, F. L., Hurdle, V. F., \& Banks, J. H. (1992). Synthesis of recent work on the nature of speedflow and flow-occupancy (or density) relationships on freeways. Transportation Research Record, 1365, pp. 12-18.

Hou, Y., Edara, P., \& Sun, C. (2015). Traffic flow forecasting for urban work zones. IEEE Transactions on Intelligent Transportation Systems, 16(4), pp. 1761-1770.

Krajzewicz, D., Erdmann, J., Behrisch, M., \& Bieker, L. (2012). Recent development and applications of SUMO-Simulation of urban mobility. International Journal on Advances in Systems and Measurements, 5(3/4), pp. 128-138.

Li, Y., Juan, C., Mori, M., \& Work, D. (2018). Estimating traffic conditions from smart work zone systems. Journal of Intelligent Transportation Systems, 22(6), pp. 490-502.

Lighthill, M. J., \& Whitham, G. B. (1955). Kinematic waves II: A theory of traffic flow on long crowded roads. In: Proceedings of the Royal Society A Mathematical Physical and Engineering Sciences, 229(1178), pp. 317-345.

Majid, H., \& Abouaïssa, H. (2015). Comparative study of a super-twisting sliding mode observer and an extended Kalman filter for a freeway traffic system. Cybernetics and Information Technologies, 15(2), pp. 141-158

Munoz, L., Sun, X., Horowitz, R., \& Alvarez, L. (2003). Traffic density estimation with the cell transmission model. In: Proceedings of the American Control Conference, pp. 3750-3755.

Newell, G. F. (1993). A simplified theory of kinematic waves in highway traffic, part II: Queueing at freeway bottlenecks. Transportation Research Part B, 27(4), pp. 289-303.

Papamichail, I., Papageorgiou, M., \& Stamatakis, I. (2018). Feedback Traffic Control at Highway Work Zones using Variable Speed Limits. In: 15th IFAC Symposium on Control in Transportation Systems, Savona, Italy, pp. 329336.

PeMS. (2016). "Caltrans Performance Measurement System." < http://pems.dot.ca.gov/>.

Qi, Y., \& Zhao, Q. (2017). Safety impacts of signalized lane merge control at highway work zones. Transportation Planning and Technology, 40(5), pp. 577-591.

Rajagopal, R.. \& Varaiya, P. (2007). Evaluating the health of californias loop sensor network. California PATH Research Report.

Richards, P. I. (1956). Shock waves on the highway. Operations Research, 4(1), pp. 42-51.

Tak, S., Woo, S., \& Yeo, H. (2016). Data-driver imputation methods for traffic data in sectional units of road links. IEEE Transactions on Intelligent Transportation Systems, 17(6), pp. 1762-1771.

Tang, J., Zhang, G., Wang, Y., Wang, H., \& Liu, F. (2015). A hybrid approach to integrate fuzzy Cmeans based imputation method with genetic algorithm for missing traffic volume data estimation. Transportation Research Part C, 51, pp. 29-40.

Yang, H., \& Rakha, H. (2017). Feedback control speed harmonization algorithm: Methodology and preliminary testing. Transportation Research Part C, 81, pp. 209-226. 